\title{
Una revisión sistemática de la morbilidad grave en los recién nacidos prematuros tardíos (1)
}

\author{
Teune MJ, Bakhuizen S, Gyamfi Bannerman C, Opmeer BC, van Kaam AH, van Was- \\ senaer AG, Morris JM, Mol BW. A systematic review of severe morbidity in infants born \\ late preterm. Am J Obstet Gynecol 2011;205:374.e1-9.
}

Análisis crítico: Juan F. Stecher M., Jorge Carvajal C., PhD.

Unidad de Medicina Materno-Fetal, División de Obstetricia y Ginecología, Pontificia Universidad Católica de Chile.

\section{RESUMEN (1)}

Objetivo: Los prematuros tardíos (34 semanas 0/7 días - 36 semanas $6 / 7$ días de gestación), representan la mayor proporción de nacimientos prematuros. Se realizó una revisión sistemática para conocer la morbilidad a corto y a largo plazo de los prematuros tardíos. Diseño del estudio: Se realizó una búsqueda electrónica de los estudios de cohorte publicados desde enero de 2000 hasta julio de 2010. Resultados: Se identificaron 22 estudios que analizaron a 29.375 .675 niños. En comparación con los recién nacidos a término, los nacidos prematuros tardíos son más propensos a sufrir peores resultados a corto plazo, tales como síndrome de dificultad respiratoria (riesgo relativo [RR]: 17,3), hemorragia intraventricular (RR: 4,9), y muerte a los 28 días (RR: 5,9). Más allá del periodo neonatal, los prematuros tardíos son más propensos a morir durante el primer año (RR: 3,7 ) y a presentar parálisis cerebral (RR: 3,1). Conclusión: Aunque la incidencia absoluta de la mortalidad neonatal y morbilidad en los recién nacidos prematuros tardíos es baja, su incidencia es significativamente mayor en comparación con los niños nacidos a término.

\section{ANÁLISIS DE LA INVESTIGACIÓN}

\section{A. Relevancia clínica de la investigación}

Los niños nacidos prematuros (antes de las 37 semanas de gestación), presentan una alta morbimortalidad. Si se excluyen las malformaciones congénitas, el $75 \%$ de las muertes perinatales y el $50 \%$ de las anormalidades neurológicas de la infancia son atribuibles directamente a la prematuridad
(2). A nivel mundial los partos prematuros han aumentado en los últimos años alrededor de un $30 \%$; en Chile este aumento es de aproximadamente un $20 \%$ entre el 2000 y 2009 (3). Este incremento ocurre principalmente entre los nacidos entre las 32 y 36 semanas, manteniéndose estables los nacimientos bajo las 32 semanas (4).

La mayoría de los esfuerzos de prevención y manejo se han concentrado en los niños nacidos bajo las 32 semanas. Sin embargo, desde el año 2005 (5) se ha realizado un llamado de atención en cuanto a que los niños nacidos entre las 34 y 36 semanas (antes conocidos como prematuros cercanos al término, hoy día como prematuros tardíos) si bien presentan menores complicaciones perinatales y mortalidad de todos los prematuros, siguen siendo prematuros y requieren un cuidado especial.

En los últimos años varios estudios han dado a conocer los resultados perinatales a corto y largo plazo de este grupo de recién nacidos, destacando la mayor frecuencia de: morbilidad perinatal (4-6), necesidades de hospitalización durante el primer mes y año de vida con mayores costos en su atención $(7,8)$, y más alta frecuencia de alteraciones neurológicas y del aprendizaje (9-11).

Conocer con precisión el pronóstico perinatal de los prematuros tardíos es indispensable en aquellas situaciones clínica que requieran la interrupción del embarazo; muchas veces se minimiza el riesgo facilitando la decisión, lo que debe ser cuestionado a la luz de los resultados que aquí se muestran.

\section{B. El estudio (1)}

El objetivo de este estudio es analizar la morbilidad severa, a corto y largo plazo, de los niños 
nacidos prematuros tardíos (34 0/7 a 36 6/7). El diseño de la investigación es una revisión sistemática de la literatura. Se buscó en las bases de datos PubMed, MEDLINE, Embase y Cochrane, todos los estudios de cohorte publicados entre enero de 2000 y julio de 2010, que comparan resultados perinatales de niños nacidos prematuros tardíos y niños nacidos a término. No hay un límite de idioma de la publicación. Se excluyen trabajos que no comparan los grupos de estudio, revisiones de la literatura, publicaciones con grupos menores de 50 niños. Los estudios fueron buscados por dos investigadores independientes, los desacuerdos fueron resueltos por consenso o por un tercer investigador. Se encuentran 314 estudios, de los cuales se lee el resumen y se descartan 266. De los 48 restantes se consigue el artículo completo y se descartan 34 por no cumplir con los criterios de inclusión y exclusión. Se analiza la bibliografía de cada uno de los artículos para identificar investigaciones nuevas que no hubieran sido encontradas con las estrategias de búsqueda señaladas, agregando 8 estudios. Se obtiene un total de 22 artículos que se analizan. Se analizaron los resultados a corto y largo plazo. El análisis estadístico se realiza compilando los datos crudos de cada uno de los estudios. Para cada resultado se calcula el riesgo absoluto (RA) y el riesgo relativo (RR), en comparación con los nacidos a término, con sus correspondientes intervalos de confianza del $95 \%$ (Tabla I).

\section{Análisis crítico}

¿Qué pregunta abordó la revisión sistemática? Este estudio busca responder a la pregunta de cuáles son los resultados perinatales a largo y corto plazo de los niños nacidos prematuros tardíos y compararlos con los niños nacidos a término.

¿Es improbable que estudios importantes y relevantes se hayan pasado por alto? Utilizan las bases de datos más importantes disponibles, no limitan la búsqueda a un idioma. Analizan la bibliografía de cada uno de los estudios seleccionados para hallar publicaciones no encontradas con las bases de datos. No señala si se contactaron con expertos o revisaron actas de los congresos más importantes de la especialidad para encontrar artículos no publicados.

¿Los criterios de selección usados para incluir a los artículos fueron apropiados? La inclusión o exclusión de estudios en la revisión sistemática estaba claramente definida desde antes de la búsqueda. Los criterios de elegibilidad usados especifican el grupo de interés. Los autores señalan los pasos de la búsqueda, como llegaron al resultado final de la búsqueda y porque descartaron algunos artículos. ¿Los estudios incluidos fueron suficientemente válidos para el tipo de pregunta? Este artículo no describe explícitamente como se evaluó la calidad de cada estudio usando, sin embargo nos parece que efectivamente la evaluación de calidad fue efectuada, puesto que descartan las publicaciones en que no es posible hacer tablas de $2 \times 2$.

¿Los resultados fueron similares entre los estudios? Los autores estiman la heterogeneidad utilizando los test Cochrane Q2, 12 y el test de Chi cuadrado. En varios de sus resultados se estima que existe heterogeneidad. En la sección de discusión del artículo se analiza como una limitación la presencia de heterogeneidad, sin embargo, se explica porque analizan a todos los nacidos prematuros tardíos, independiente de la causa de la prematuridad o de las condiciones obstétricas. Pese a existir heterogeneidad, todos los riesgos relativos son de la misma dirección del riesgo $(R R>1)$.

¿Cuáles fueron los resultados y cómo se presentan? Los autores incluyeron 22 estudios que cumplieron los criterios de inclusión, con un total de 2.368.471 prematuros tardíos y 27.007.204 nacidos de término. Los resultados de corto plazo se presentan en tablas, calculando el riesgo relativo absoluto, agrupados para el grupo de prematuros tardíos en general y por edad gestacional, comparando con el grupo de nacidos a término. Se presentan dos gráficos de bosque en que se compara la mortalidad neonatal e infantil del grupo de ambos grupos de estudio. La morbilidad a largo plazo es descrita en el artículo, pero no es presentada como tabla o gráfico de bosque. El resultado de la síntesis de los principales resultados se resumen en la Tabla I.

La mortalidad infantil, es casi 4 veces más frecuente entre los nacidos prematuros tardíos que en los de término (RR: 3,7; IC95\% 2,9-4,6; RA: 0,83\% vs $0,27 \%$ ). Los prematuros tardíos tienen más riesgo de presentar parálisis cerebral que los niños de término (RR: 3,1; IC95\% 2,3-4,2; RA 0,43\% vs $0,14 \%)$, y más riesgo de desarrollar retardo mental (RR: 1,5; IC95\% 1,2-1,9; RA: 0,81\% vs 0,49\%). En etapa escolar los prematuros tardíos tienen más riesgo de presentar un retraso en el desarrollo y problemas escolares que los niños de término, durante los primeros 5 años de vida.

Conclusión de los autores. Aunque la incidencia absoluta de mortalidad neonatal e infantil y complicaciones a corto y largo plazo es baja en los niños nacidos prematuros tardíos, esta incidencia es significativamente más alta que en los niños nacidos a término. Esta información debería ser tomada en cuenta para el manejo de las mujeres con alto riesgo de un parto cercano al término. 


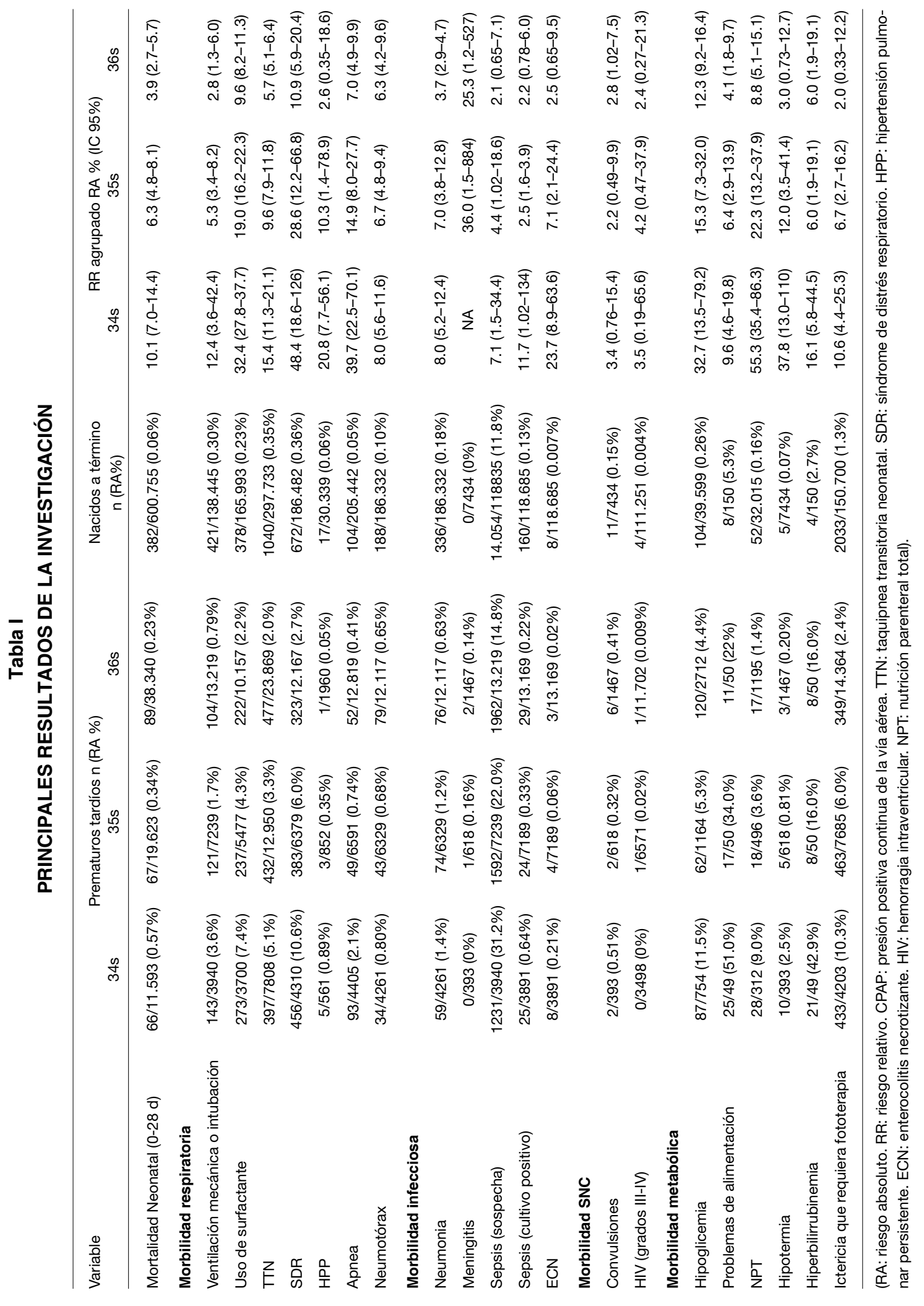


Comentarios del análisis. Revisión sistemática de alta calidad metodológica con procedimientos estándar para minimizar el riesgo de sesgo en la selección de los estudios, la extracción de datos y el análisis. Sólo incluye estudios de cohorte, con las desventajas que ellos presentan. Sin embargo, se estima que la posibilidad de cambiar las conclusiones actuales es baja. Las conclusiones de los autores reflejan el análisis realizado. Recomendamos la inclusión de la información presentada en este artículo durante la toma de decisiones de interrupción del embarazo por patología materna o fetal entre las 34-36 semanas.

\section{REFERENCIAS}

1. Teune MJ, Bakhuizen S, Gyamfi Bannerman C, Opmeer BC, van Kaam AH, van Wassenaer AG, Morris $\mathrm{JM}$, Mol BW. A systematic review of severe morbidity in infants born late preterm. Am J Obstet Gynecol 2011;205:374.e1-9.

2. Goldenberg, RL, Culhane, JF, lams, JD, Romero, R. Epidemiology and causes of preterm birth. Lancet 2008;371:75-84.

3. González R, Nien JK, Vera C, Poblete JA, Carvajal J, González M, et al. ¿Existe un aumento de los nacimientos en Chile en el período 2000-2009?: Análisis de los principales indicadores materno-infantiles de la década. Rev Chil Obstet Ginecol 2011;76:404-11.

4. Davidoff MJ, Dias T, Damus K, Russell R, Bettegowda
VR, Dolan S, Schwarz RH, Green NS, Petrini. Changes in the gestational age distribution among U.S. singleton births: impact on rates of late preterm birth, 1992 to 2002 .Semin Perinatol 2006;30:8-15. Erratum in: Semin Perinatol 2006;30:313.

5. Raju TN, Higgins RD, Stark AR, Leveno KJ. Optimizing care and outcome for late- preterm (near-term) infants: A summary of the workshop sponsored by the National Institute of Child Health and Human Development. Pediatrics 2006;118:1207-14.

6. Wang ML, Dorer DJ, Fleming MP, Catlin EA. Clinical outcomes of near-term infants. Pediatrics 2004;114:372-6.

7. McLaurin KK, Hall CB, Jackson EA, Owens OV, Mahadevia PJ. Persistence of morbidity and cost differences between late-preterm and term infants during the first year of life. Pediatrics 2009;123:653-9.

8. Underwood MA, Danielsen B, Gilbert WM. Cost, causes and rates of rehospitalization of preterm infants. $J$ Perinatol 2007;27:614-9.

9. Petrini, JR, Dias, T, McCormick, MC, et al. Increased risk of adverse neurological development for late preterm infants. J Pediatr 2009; 154: 169.

10. Chyi, LJ, Lee, HC, Hintz, SR, et al. School outcomes of late preterm infants: special needs and challenges for infants born at 32 to 36 weeks gestation. J Pediatr 2008; 153:25.

11. Moster, D, Lie, RT, Markestad, T. Long-term medical and social consequences of preterm birth. $\mathrm{N}$ Engl $\mathrm{J}$ Med 2008; 359:262. 